came from home (57\%). Overall, 39\% died. Mortality varied by race $(\mathrm{p}<0.01)$ : white $39 \%$, black $39 \%$, Hispanic $31 \%$, other races $51 \%$. In the logistic regression model, age, race, and residence were significant predictors of mortality, after adjustment for other variables. Each additional year of age had a $2.7 \%$ increased odds of mortality (OR 1.03; 95\% CI 1.02-1.03; $\mathrm{p}<0.01$ ). Compared to white patients, odds of death were 1.6 times higher for other races $(95 \%$ CI 1.3 2.0; $\mathrm{p}<0.01$ ) and non-significantly higher for black patients (OR $1.1 ; 95 \%$ CI 1.0-1.2; $\mathrm{p}=0.05)$. Compared to those from home, odds of death were highest for those from a skilled nursing facility (OR 1.5; $\mathrm{p}<0.01)$. DISCUSSION/SIGNIFICANCE OF IMPACT: Patients who identified as other races had increased mortality from septic shock compared to white patients after adjusting for other variables. Septic shock mortality also increased with age and varied by residence. Further analyses are needed to examine racial disparities and control for comorbidities, severity of illness, and aspects of resuscitation. CONFLICT OF INTEREST DESCRIPTION: The authors report no conflicts of interest, except for Dr. Fernandez, who reports personal payment from Physio-Control, Inc. for speaker fees.

4433

SGRQ score is associated with treatment status for patients with non-tuberculous mycobacterial lung disease

Bryan Garcia ${ }^{1}$, Abigail Grady ${ }^{2}$, Lilian Christon², Patrick Flume², and Susan Dorman²

${ }^{1}$ Medical University of South Carolina; ${ }^{2}$ MUSC

OBJECTIVES/GOALS: The Saint Georges Respiratory Questionnaire (SGRQ) is used as a patient reported outcome tool for clinical research in COPD and bronchiectasis. We established a registry and biospecimen repository of bronchiectasis patients with and without NTM and report associations between clinical phenotype and SGRQ scores. METHODS/STUDY POPULATION: Patients were recruited in a cross-sectional format from the Bronchiectasis, Cystic Fibrosis, and NTM clinics at our institution. All patients provided at least one sputum sample in the six months prior to inclusion. Clinical and epidemiologically relevant data was obtained, and blood specimens were processed and preserved. Patients were grouped based on clinical phenotype and differences in SGRQ scores were analyzed using ANOVA or Student's t-test. Descriptive statistics are reported as means and standard deviations, $\mathrm{p}<0.05$ considered significant. RESULTS/ANTICIPATED RESULTS: 72 NTM patients completed the SGRQ including 39 patients not on treatment (Colonized), 29 patients on NTM directed antibiotics, and 4 patients whose infection was cured in the past year. Among patients on treatment, 14 were treatment refractory (positive cultures beyond 12 months of therapy). The mean age of all NTM patients was $59.5 \pm 17.6$ and $80.5 \%$ were female. Mean SGRQ Total scores were significantly higher among patients receiving treatment compared to patients considered colonized (35.7 \pm 22.0 colonized group versus $48.8 \pm 15.8$ treatment group, $\mathrm{p}=0.011)$. The SGRQ subdomain scores including Impacts $(26.2 \pm 26.2$ colonized group versus $42.5 \pm 17.0$ treatment group, $\mathrm{p}=0.01)$ and Activities $(41.7 \pm 31.8$ colonized group versus $59.3 \pm 24.5$ treatment group, $\mathrm{p}=0.018)$ were also significantly different between groups. DISCUSSION/ SIGNIFICANCE OF IMPACT: We developed a cross sectional cohort of NTM patients and assessed associations between clinical phenotype and SGRQ score. Preliminary data suggests that female sex, treatment status, and therapeutic duration are associated with higher SGRQ scores. We intend to continue to assess the potential for specific SGRQ questions to be used for quantifying disease symptom severity for NTM patients.

4236

\section{Sleep Disorders and Diabetic Complications}

Magda Shaheen ${ }^{1}$, Meleesa Nocera ${ }^{2}$, and Senait Teklehaimanot ${ }^{2}$ ${ }^{1}$ David Geffen School of Medicine at UCLA; ${ }^{2}$ Charles R Drew University

OBJECTIVES/GOALS: Diabetes is a prevalent chronic illness that imposes health-related burdens including nephropathy, retinopathy and sleep disorders. The goal of this study was to examine the relation between both sleep disorders and sleep duration and diabetic chronic kidney disease (CKD) and retinopathy. METHODS/ STUDY POPULATION: We analyzed data from the National Health and Nutrition Examination Survey 2005-2016 related to diabetic nephropathy and retinopathy, sleep disorders and duration, demographics, and risk factors among diabetics. The subjects were adults with diabetes type 2. Multiple logistic regression analysis was performed to look at the relationship between diabetic complications (CKD and retinopathy) and sleep disorders and sleep duration adjusting for demographics and risk factors. RESULTS/ ANTICIPATED RESULTS: Of the 4087 diabetics, 45\% had CKD, $19 \%$ had retinopathy, and $15 \%$ had sleep disorders. CKD and retinopathy were not associated with sleep disorders $(p>0.05)$ but CKD was associated with sleep duration (Adjusted odds ration $=1.014,95 \%$ confidence interval $=1.001-1.027, \mathrm{p}<0.05)$. Cardiovascular disease was a predictor of both CKD and nephropathy $(\mathrm{P}<0.05)$. Other predictors of $\mathrm{CKD}$ and nephropathy were age $>60$ years, Non-Hispanic Black, hypertension, low education level, and living under $200 \%$ of the Federal Poverty Level $(\mathrm{P}<0.05)$. DISCUSSION/SIGNIFICANCE OF IMPACT : Among diabetics, $\mathrm{CKD}$ and retinopathy were not associated with sleep disorders, and only CKD was associated with sleep duration. These findings may impact the management of diabetes in the future, since it has effects on a range of other health conditions.

4327

Structural Neural Correlates of Social Functioning in First Episode Psychosis and Malleability in Response to Targeted Cognitive Training

Kathleen Miley ${ }^{1}$, Fang Yu, Ian Ramsay, and Sophia Vinogradov

${ }^{1}$ University of Minnesota CTSI

OBJECTIVES/GOALS: Development of interventions that improve social functioning (SF) in first episode psychosis (FEP) is hindered by a poor understanding of the neural mechanisms underlying SF deficits. This research aims to identify neural correlates of social functioning in FEP, and to evaluate whether this substrate is malleable in response to cognitive training. METHODS/STUDY POPULATION: This is a secondary data-analysis of participants in an ongoing randomized clinical trial investigating whether 12 weeks of targeted cognitive training is neuroprotective in FEP, versus treatment as usual. Baseline and post-training assessments include a brain MRI, three 